\title{
Mental Health and Psychosocial Implications on the Medical Workers during the Pandemic COVID-19 Outbreak in Pakistan: An Empirical Review
}

\author{
Abdul Rahim Chandio ${ }^{1^{*} \text { (D) }}$ \\ ${ }^{1}$ University of Sindh, Pakistan \\ * Corresponding author: Abdul Rahim Chandio E-mail: chandiorahim@gmail.com ORCID: 0000-0001-8889-8780 \\ Received: 21 May 2020 Accepted: 14 June 2020
}

\begin{abstract}
The current study provides an overview of the COVID-19 implications on the health workers by means of their mental health and psychosocial aspects. It develops the sense of fear and susceptibility of the health workers for coping the strategies and medical targets and such perceived stress remained so fatal and harmful to the workers psyche to meet the job satisfaction under the prevailing situation due to the outbreak of the pandemic COVID-19. The prevention measures failed and became a futile attempt to seize the atmosphere of stressfulness for the frontline workers to combat the COVID-19 and it returned in the form of fear, anxiety and emotional dilemmas of isolation and loneliness. This study assists to investigate perception of the medical workers by empirical means either they face psychosocial worriness and mentally stress relating to their service during the COVID-19 outbreak in Pakistan. For the data collection, an online survey was conducted from 30 April to 20 May in which 392 questionnaires were distributed among Pakistani doctors and paramedical staff and the response rate remained 256 (65.3\%) to discover their concern on the rising dilemma of the COVID-19. For the empirical analysis, proposed variables were adopted to carry out the study in order to find out the intentional level of the health workers regarding their mental distress and psychosocial problems. For the data collection, respondents were participated from the Sindh province of Pakistan in which data was analyzed through SPSS version 24 as it applied the regressions and Pearson's correlation to test the hypothesis that resulted to reveal all factors to sustain the significant positive relationship with dependent construct.
\end{abstract}

Keywords: Pakistan, COVID-19, implications, mental health

\section{INTRODUCTION}

An overview concerning the implication of the COVID-19 outbreak on the health workers in the form of mental health and psychosocial aspects that allow the emergence of the sense of strong fear, perceived threat, and stress appraisal in the way of managing the provision of the health initiatives to the public during an outbreak of COVID-19. Prevention remains a key element in the managing, controlling, and monitoring of the COVID-19 pandemic in Pakistan. A person suspected or infected with COVID-19 pandemic follow the practices of self-isolation and social distancing at home, or pursues the medical care facility with infrastructure for treating COVID-19 [1]. The problems relating to mental health can occupy and aggravate psychological stress, frustration, and emotional distress due to self-isolation and 
an individual's quarantine [1-2]. The medical health-care workers remain in direct contact with the COVID-19 patients either ill person is confirmed or under the suspected case that represents a scenario of vulnerability and high-risk infection which leads to mental health problems for instance stress, trauma, and experiencing bereavement. With the emergence of the COVID-19 pandemic in Pakistan, health workers face the physical and psychological pressure that encompasses a high risk of infection, scarcity of safetyrelated equipment from contagion, isolation, exhaustion, and lack of contact with the relatives or family members [3]. The prevailing pandemic has erupted the psychological stress in the society and with the advent of Coronavirus (COVID-19) is on high alert. The increasing social isolation, stress, health anxiety, loneliness, and an economic downturn are a perfect storm not only harms to health workers and the general public's mental health and wellbeing. During the outbreak of pandemic COVID-19, psychological crisis intervention for affected, suspected, susceptible, and at-risk patients, caregivers, families, health staff, and the general public, is urgently needed for timely prevention of inestimable hazards from the secondary mental health crisis.

The premise of psychological crisis intervention is to control the side- and after-effects on psychosocial aspects of infectious disease and attempt to minimize psychological impact with timely assessment and management of prevention and control [4]. The implication of the rising COVID-19 has deeply impacted the glob mentally, and economically as Pakistan is also victimized likely other nations of the world. The pandemic not only became the cause of a high mortality rate due to the viral infection and it also brought a catastrophe of a psychological rest and mental devastation in the rest of the world [5]. The severity remains an additional mental health dilemma that affects the medical workers and their decision-making capacity and it can also possess a detrimental effect on their overall longterm well-being. Due to intensive stress of the medical health workers is experiencing to encircle the psychological issues of fear, anxiety, posttraumatic stress, depressiveness, sleep disturbances, avoidance of contacts, social isolation from the family and loved one, helplessness, and psychological anxiety [12-23].

A sudden change in the status of a healthcare provider or health frontline face the psychological and mental problems due to the COVID-19 case to be confirmed or suspected patients in the form of frustration, helplessness, and difficult to adopt the healthcare professionals and the fear of labeling, stigma, and discrimination can be a deterrent and medical healthcare soldiers envisage the counseling and psychotherapeutic involvements [10-23]. Despite the usual mental health issues and the problems concerned with psychosocially of professionals' health care workers in general, most health professionals often do not seek or receive mental health care [5]. Medical professionals and health care workers who perform their work under severe stress possess the behavioral and emotional responses naturally adapted to extreme stress, and therefore modelbased on counseling and psychotherapy can act on stress factors as soon as early and timely intervention [12-14].

\section{COVID-19 IMPLICATIONS ON MEDICAL WORKERS AND MEASURES}

The COVID-19 outbreak is a pandemic in which it has been identified as the cause of an outbreak of respiratory illness. The primarily transmission and spreading of the virus is based on social contact usually by means of the mobility of small droplets shaped by sneezing, coughing, and talking. The pandemic was detected in Wuhan, China firstly [8-9] but COVID-19 spread to become a global dilemma and a rapid pandemic in a very shortly [8]. Pakistan is a third world nation and it cannot sustain the long live social, political, economic, and psychological stress and deprivation as prevailing in the public health workers and general masses. Therefore, solving the mental health problems of health workers is important for better prevention and control of the COVID-19, pandemic [11].

The medical workers are usually in work routinely to care for the patients of the COVID-19, under the condition of their confirmed and suspected cases can increase the pressure on healthcare personnel. In this case, online and electronic media can provide medical advice on how to prevent the risk of transfer between patients and clinicians who may reduce the burden on medical professionals. The critical conditions require an effective plan to impede the psychological catastrophe as a comprehensive plan for intervention for mental disorders needs to be improved.

- By developing a team to intervene the mental health issue and psychological problems via giving online courses to enlighten them regarding the psychological implications and its stressful behavior on the health workers.

- By providing a guiding booklet and conducting seminars and training to medical workers to defend the situations in a dynamic way in which experiences of the COVID-19 affected nations must be shared. 
- Adopting technological use must be preferred and monetary incentives encouraged timely in order to boost up the health workers' confidence accordingly.

- Ensuring the provision of psychological assistance to health workers and encouraging them to discuss the psychological problems to trained and experienced.

- Developing strong leadership with deep clarity, rationality, honesty, and open communication is required to encounter the fears and the atmosphere of uncertainties.

- The provision of adequate resources or medical equipment and mental health supports initiatives can encourage medical worker's confidence and selfefficacy.

The medical Health workers may face substantial psychological distress due to the COVID-19 pandemic by giving direct patient care, vicarious trauma, quarantine, or self-isolation [12-14]. The adoption of online technology can assist and encourage confidence to be secure from the risk as using online technology allows providing psychological support by keeping physical distance [13]. The emphasis on altruism in healthcare and the service of excellent products helps healthcare professionals to remind themselves of their goals during times of crisis. Hospital units should be given frequent shift-system, living supplies, guarantee food, and provide pre-job training to deal with to find out and react to psychological issues. Additionally, counseling with psychologists on a regular basis can assist the medical workers to comprehend their concerns and offer their support in this regard.

\section{THEORETICAL FRAMEWORK}

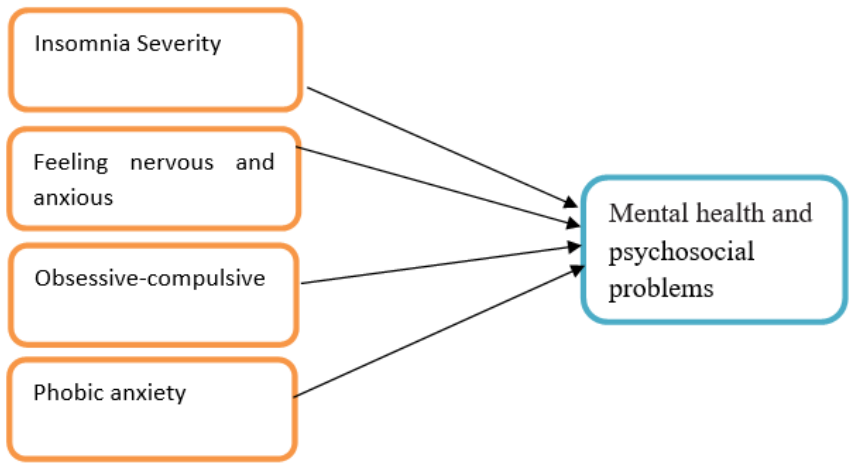

Figure 1. Conceptual framework model

\section{HYPOTHESIS}

- There is a significant positive relationship between Insomnia Severity and mental health and psychosocial problems.

- There is a significant positive relationship between Feeling nervous and anxious and mental health and psychosocial problems.

- There is a significant positive relationship between Obsessive-compulsive symptoms and mental health and psychosocial problems.

- There is a significant positive relationship between Phobic anxiety and mental health and psychosocial problems.

The above-proposed variables relationship in the hypotheses reveal that all independent constructs maintain a positive relationship with the dependent variable. In this regard, it suggests that positive significant association of theses hypotheses highlight that all psychological stressrelated factors encourage mental health and psychosocial problems.

\section{CONCEPTUALIZATION OF VARIABLES}

The Insomnia Severity implies a brief instrument that was designed to assess the severity of both nighttime and daytime components of insomnia [12-15]. Insomnia gives the symptoms like difficulty falling asleep at night, Waking up during the night, Waking up too early, not feeling wellrested after a night's sleep, daytime tiredness, irritability, depression, or anxiety, difficulty paying attention, focusing on tasks or remembering and increased errors or accidents. Mental health and psychosocial problems mention to extreme stressors and mental health issue as common mental disorders as it is related to the circumstance. The risk indicators including chronic insomnia as a factor to highlights that increase of the COVID-19 with contact to lead insomnia, depression, anxiety, and obsessive-compulsive symptoms among medical health workers [16]. Participants in the existing study discover whether they face insomnia or psychiatric disorders in their services to given in hospitals and they replied positively having the implications of insomnia during their service in the COVID-19 outbreak. The factor Feeling nervous/anxious was also measured earlier researcher to discover the empirical investigation of the medical health worker during their work COVID-19 pandemic to make health providers worry and emotionally nervous [12-21]. 
Table 1. Demographic Characteristics

\begin{tabular}{|c|c|c|c|}
\hline Variables & Description & Frequency & Percentage \\
\hline \multirow{2}{*}{ Gender } & Male & 242 & 94.5 \\
\hline & Female & 14 & 5.4 \\
\hline \multirow{4}{*}{ Age } & $21-30$ & 20 & 7.8 \\
\hline & $31-40$ & 170 & 66.4 \\
\hline & $41-50$ & 27 & 10.5 \\
\hline & $51-60$ & 39 & 15.2 \\
\hline \multirow{2}{*}{ Marital Status } & Single & 106 & 42.1 \\
\hline & Married & 148 & 57.8 \\
\hline \multirow{3}{*}{ Education } & Bachelor & 79 & 30.8 \\
\hline & Master & 130 & 50.7 \\
\hline & M.Phil/ P.hd & 47 & 18.3 \\
\hline \multirow{4}{*}{ Job Experience } & Less than 1 year & 29 & 11.3 \\
\hline & $1-5$ years & 103 & 40.2 \\
\hline & 6-10 years & 87 & 33.9 \\
\hline & $11-15$ years & 37 & 14.4 \\
\hline
\end{tabular}

Obsessive-compulsive present the unwanted thoughts and fears (obsessions) which lead to confusion and significant distress, anxiety, bothersome, and the emergence of an excessive fear to be infected with illness and getting contaminated by germs and symptoms were assessed [1218]. Moreover, the previous studies were also measured the proposed factors for instance Somatic symptoms, obsessivecompulsive symptoms, and phobic anxiety [17-18]. During the outbreak of the COVID-19 epidemic, medical health workers had to face the severe condition of insomnia, obsessive-compulsive symptoms, depression, anxiety, and somatization. The medical workers had risk antecedents for rising obsessive-compulsive, insomnia, somatization, anxiety, and depression. Henceforth, the prevalence of these types of symptoms in the daily life basis to fight against the COVID-19 pandemic recommends coping the psychological distress and diminishing the workload and ensuring the provision of medical infrastructure and progressive counseling [12-20].

Phobic anxiety becomes the cause of a person's to experience extreme irrational fear to consider being dangerous [12]. The Phobic anxiety was measured by the various studies to investigate the extreme irrational fear of the medical worker $[17,18]$. According to sources that the frontline role provider doctors and nurses serve the COVID19 patients as the number of the health soldiers are admitting to harboring darker feelings, frustration, anxiety, fear, and psychological distress [23].

\section{RESULTS AND DISCUSSION}

The proposed factors and their hypothesis maintain the significance of independent constructs with dependent factor as insomnia severity, feeling nervous, obsessivecompulsive symptom, and Phobic anxiety with mental health and psychosocial problems. In this way, all hypotheses represent the positive impact of the independent variable to increase the mental health and psychosocial problems as results reveal. Moreover, these factors were used by the earlier researcher [12-21] whereas in the current study theses variables have been adopted slightly dynamically in the way to investigate the rising mental health and psychosocial problems of the medical workers in the prevailing critical scenario of the COVID-19. For the data collection five points Likert scale has been used in which five option were given to choose like (1) strongly disagree (2) Disagree (3) neutral (4) agree and (5) strongly agree as in the earlier researcher also applied it with a similar type of the study [17-21]. The existing study pursues the correlation and regression analysis in order to test hypotheses validity.

It is important to verify the multivariate correlation to examine the relationship between the variables. Thus, researchers investigate the independent variables and the dependent variable's relationship through Pearson's Correlation. Results indicate a significant relation at the level of 0.1 whereas all variables of the conceptual model supported and remained insignificant positively correlated with the criterion variable.

\section{Correlation}

The correlation in this study encompasses and remains between all factors as (.473 to .598) whereas correlation of the IS (.597), FNA (.576-.507) OC (.258-.260) and PA (.571-.185) confirmed. The correlation can be highlighted in 
Table 2. Survey items, standard deviations and mean

\begin{tabular}{|c|c|c|}
\hline Item statement (short description in English) & Mean & SD \\
\hline Q1: Falling asleep (Insomnia Severity) & 3.691 & .9643 \\
\hline Q2: I expect to early awakening (Insomnia Severity) & 3.7468 & .85112 \\
\hline Q3: I remained worried (Insomnia Severity) & 3.7124 & .86536 \\
\hline $\begin{array}{l}\text { Q4: the rising COVID-19 pandemic creates psychological stress for me to work in a hospital (mental health and } \\
\text { psychosocial problems) }\end{array}$ & 3.6652 & .84560 \\
\hline $\begin{array}{l}\text { Q5: I remain in fear and confusion due to inadequate medical equipments to me that lead me towards mental } \\
\text { health issues (mental health and psychosocial problems) }\end{array}$ & 3.7210 & .76245 \\
\hline $\begin{array}{l}\text { Q6: The inadequate facility, isolation from the family, decline of monetary incentives, and rising cases of the } \\
\text { COVD-19 patients encourage my anxiety and psychological distress. (mental health and psychosocial problems) }\end{array}$ & 3.7897 & .72723 \\
\hline Q10: I face and consider Unwanted thoughts/words and Feeling blocked (obsessive-compulsive). & 3.7768 & .78344 \\
\hline $\begin{array}{l}\text { Q11: In the critical situation of the COVID-19 I judge myself to do very slowly and face trouble remembering } \\
\text { things (obsessive-compulsive). }\end{array}$ & 3.8155 & .77422 \\
\hline Q12: I consider trouble concentrating and Worried about sloppiness (obsessive-compulsive). & 3.8541 & .73999 \\
\hline Q13: I face difficulty in making decisions (obsessive-compulsive). & 3.8412 & .79091 \\
\hline Q14: I am in a situation to afraid in open space and travel (phobic anxiety) & 3.7124 & .74201 \\
\hline Q15: I am in a condition to avoid certain and feeling uneasy in crowds (phobic anxiety). & 4.0043 & 2.68215 \\
\hline Q16: I am in a nervous situation when left alone and Afraid to faint in public (phobic anxiety). & 3.8155 & .76300 \\
\hline
\end{tabular}

Table 3. Pearson correlation

\begin{tabular}{|c|c|c|c|c|c|}
\hline & MHPP & IS & FNA & OC & PA \\
\hline Mental health and psychosocial problems (MHPP) & 1 & & & & \\
\hline Insomnia Severity (IS) & $.598^{* *}$ & 1 & & & \\
\hline Feeling nervous and anxious (FNA) & $.586^{* *}$ & $.507^{* *}$ & 1 & & \\
\hline Obsessive-compulsive (OC) & $.541^{* *}$ & $.497^{* *}$ & $.473^{* *}$ & 1 & \\
\hline Phobic anxiety (PA) & $.562^{* *}$ & $.572^{* *}$ & $.556^{* *}$ & $.534^{* *}$ & 1 \\
\hline
\end{tabular}

${ }^{* *}$. Correlation is significant at the 0.01 level (2-tailed).

Table 4. Regression analysis

\begin{tabular}{|c|c|c|c|c|c|c|}
\hline \multicolumn{7}{|c|}{ Coefficients } \\
\hline & \multirow{2}{*}{ Model } & \multicolumn{2}{|c|}{ Unstandardized Coefficients } & \multirow{2}{*}{$\begin{array}{c}\text { Standardized Coefficients } \\
\text { Beta }\end{array}$} & \multirow{2}{*}{$\mathbf{T}$} & \multirow{2}{*}{ Sig. } \\
\hline & & B & Std. Error & & & \\
\hline \multirow{5}{*}{1} & (Constant) & -.799 & .289 & & -2.687 & .008 \\
\hline & IS & .382 & .093 & .283 & 4.670 & .000 \\
\hline & FNA & .343 & .098 & .255 & 4.326 & .000 \\
\hline & OC & .267 & .089 & .198 & 3.435 & .001 \\
\hline & PA & .217 & .093 & .153 & 2.431 & .016 \\
\hline
\end{tabular}

a. Dependent Variable: MHPP

Insomnia Severity (IS) (.598) whereas the lowest Obsessivecompulsive $(\mathrm{OC})$ is in (.473). The multiple regression analysis assists to find out the effect of independent constructs with the dependent factor whereas the regression table tests the hypothesis as presented below.

The research methodology is based on the quantitative data analysis of an online survey. The survey questionnaires could assist in the analysis of data and follow the frequencies and respondents' profiles. Moreover, for the data analysis, SPSS was used to comprehend the relationship of the factor and reliability of the factor was discovered in order to meet the level measure of internal consistency and reliable scale whereas the linear regression presents a Statistical Data Analysis to determine the relationship between a dependent factor to independent variables and the use of the Pearson Correlation assist to test a statistically significant relationship. The further prevailing study interprets that the relationship of all independent variables to dependent construct positively associate which shows that the constructs as IS, FNA,OC and PA increase the anxious and mental health and psychosocial problems (MHPP) level of the medical worker during the COVID-19 pandemic outbreak. 


\section{RISK MANAGEMENT AND SUGGESTIVE MEASURES}

The rising emergence of fear, anxious and mental health and psychosocial problems require well-being to introduce the risk management measures in order to turn down the rising dilemma. Integrating mental health into primary and community care to meet basic health needs and health workers face the psychological distress as they ensure the provision of services to direct patient care or self-isolation (quarantine) of the COVID-19 pandemic patients require adequate medical infrastructure and equipment. The government must ensure the provision of the psychological first aid in emergency situations and resources must be applied to identify and protect the vulnerable groups.

curbing the misuse of media to spread the fake rumors in order create the fear and anxiety that lead to psychological stress and mental health issues and engage people to facilitate self-sufficiency and propagating timely information regarding the emergency. Adoption of the digital system can assist in the medical science to share the experience and it can also socialize the health workers from the earlier how to deal the situation and development of mental health and psychological relief can be promoted via online mobility of the encouragement and transmission of the initiatives [13]. Likely, the researcher argues rapid development and evaluation process can be accessed through electronic package (e-package) to maintain the psychological wellbeing of the medical workers or health providers during and after the COVID-19 pandemic [13].

\section{IMPLICATIONS FOR RESEARCH AND PRACTICES}

The findings in this study implies to assess the psychological stress of the medical health workers during COVID-19 outbreak in Pakistan that urges the government to raise the psychology-related canselling and encouraging public awareness to motivate the frontline workers via their salutations in order to build faculty of trust and confidence. Such psychological and mental health-related implications call for more efforts by the Pakistan government via adopting the effective strategy, ensuring the feedback with credibility to service providers. A call of a government support in the form of providing enough medical infrastructures, investment of adequate GDP on the public health, establishing more medical universities and researcher centers in the diverse venues across the country in order to address the health of people on except any discrimination. Researchers explore that rising stress of the health workers in the prevailing COVID-19 outbreak needs to develop initiatives to materialize reliability and assist to incorporate the social factors to discover the people's opinion regarding the workers in order to evaluate the mental health and psychosocial problems.

This research occupies a significance to discover the degree of stress of the doctors or paramedical staff in a Pakistan perspective and finding confers the independent variables' significant relationship with dependent factor (MHPP) that argue the high level of the psychological distress prevails of the workers. It is represented the importance of the determinants ( $\mathrm{SI}$ beta value $=.0283, \mathrm{FNA}=.255, \mathrm{OC}=.198$, and $P A=.153)$ as these factors influence the dependent variable to lead a positive significant relationship related to psychological suffering. The implications of this study provide a discussion to investigate the mental health and psychosocial effects on the medical workers during the pandemic COVID-19 outbreak in Pakistan. The results of the extant study can urge the government to devise the measures to meet the rising stress of the health frontline soldiers in the country and apply the international experiences to motivate the health workers to encounter the COVID-19 pandemic in an effective and efficient means and they can maximize their services with limited capacity and medical infrastructure to combat the COVID-19 pandemic and encourage people to defeat the dilemma as a brave nation that can result to weaken the erupting volcano of psychological distress and extreme fear in the society. The primary implication in this study encircles the significance based on constructing a theoretical conceptual framework in order to carry out the empirical means investigation of the mental health and psychological stress of the health workers by an online survey.

\section{LIMITATIONS AND FUTURE DIRECTION}

The existing study is based on the online survey and in a cross-sectional nature. The data collection and respondents' feedback followed the voluntary online participation of the medical health workers in the various hospitals across the Sindh province. Moreover, collection of the data was gathered from medical health workers which was carried out under the severe and hard circumstances which can encourage more stress as compared to the common degree of anxiety of a work that requires re-investigation and it urges to distribute and secure more survey questionnaire up to 1200 samplings to prove the fact in a perfect means in future due to dynamic traits of human behavior.

The extant study used the newly instrument (online survey questionnaires' items) written in English to achieve respondents' minds feedback as they work in different hospitals (Larkana, Karachi, Sukkar, Mirpur, Hyderabad, Nawabshah) of Sindh. And data was collected from the 
major hospitals of (Liaquat university of medical and health sciences, Taluka hospital Hyderabad, Isra university hospital, Agha Khan, Indus Hospital Karachi, Chandka Medical College/Arija campus, Taluka hospital Mehar Dadu, Railway Hospital Sukkur, JIMS Jacobabad, Taluka Hospital of Nasirabad, and Tulka hospital K.N shah) in which respondents privacy strictly was maintained. This study is concerned with sample of the medical health workers focusing on their stress during the COVID-19 outbreak as the medical workers provided their participation as they were working in their diverse fields or specialties to serve people in their respective hospitals.

It is recommended for future researchers to use clinical interviews to portray a more comprehensive evaluation of the dilemma. The coming researcher can apply the model for measuring the medical health workers' stress in all other services to be provided to patients in their different illnesses besides COVID-19 patients. The crucial role of the health providers can be defined as the protector of humanity in this process of the difficult COVID-19 outbreak to develop a more enlightened, attentive, and theoretical based perspective to position of their practice and research. The COVID-19 can affects an individual and communities with a negligible privileges thus it is crucial to envisage an strategy to implement the scheduled assignments and provide services to people which require to assess the issues and concern of the medical health workers in order to draw up and execute an effective service delivery and producing an appropriate security measures to health providers via materializing psychological relief and degree of reliability and confidence to carry out the task in hand.

\section{CONCLUSION}

The rising crises of the COVID-19 present the miserable condition of the national health system in Pakistan, and the developed world which brought the harmful implication in the lives of the medical workers in the form of social stress, and psychological distress. During the outbreak of COVID19 , healthcare workers had psychosocial problems and risk factors for their development which require assistance and deep care of the government with adequate health facilities and medical preventive equipment. In the results of the present study is to encompass the positive relationship of all independent variables and dependent variable in which the proposed construct encourages the mental health and psychosocial problems. Covid-19 is not only detrimental to the lives of patients but also the lives of medical frontline providers, as numerous healthcare workers who are leading the battle worldwide. Pakistan is also under increasing pressure as medical workers play a key role and serve as frontline soldiers whereas rising psychological stress is a serious challenge to sustain demand and supply in the way to ensure the provision of the medical facility to people.

\section{DECLARATION OF CONFLICT OF INTEREST}

The authors received no financial support for the research and/or authorship of this article. There is no conflict of interest.

\section{REFERENCES}

1. Mukhtar S. Mental health and emotional impact of COVID-19: Applying Health Belief Model for medical staff to general public of Pakistan. Brain, Behavior, and Immunity. 2020; 87: 28-29. (doi: 10.1016/j.bbi.2020.04.012).

2. Kelvin DJ, Rubino S. Fear of the novel coronavirus. J. Infect. Dev. Countries 2020; 14(1): 1-2. (doi: 10.3855/jidc.12496).

3. Rana W, Mukhtar S, Mukhtar S. Mental health of medical workers in Pakistan during the pandemic COVID-19 outbreak. Asian journal of psychiatry. 2020: 51: 102080. (doi: 10.1016/j.ajp.2020.102080).

4. Kang L, Li Y, Hu S, Chen M, Yang C, Yang BX, et al. The mental health of medical workers in Wuhan, China dealing with the 2019 novel coronavirus. The Lancet Psychiatry, 2020; 7(3): e14. (doi: 10.1016/S22150366(20)30047-X).

5. Xiao C. A novel approach of consultation on 2019 novel coronavirus (COVID-19)-related psychological and mental problems: structured letter therapy. Psychiatry investigation, 2020; 17(2): 175-176. (doi: 10.30773/pi.2020.0047).

6. Kang L, Ma S, Chen M, Yang J, Wang Y, Li R, et al. Impact on mental health and perceptions of psychological care among medical and nursing staff in Wuhan during the 2019 novel coronavirus disease outbreak: A crosssectional study. Brain, behavior, and immunity. 2020; 87: 11-17. (doi: 10.1016/j.bbi.2020.03.028).

7. Montemurro N. The emotional impact of COVID-19: From medical staff to common people. Brain, behavior, and immunity. 2020; 87: 23-24. (doi: 10.1016/j.bbi.2020.03.032).

8. Lancet T. COVID-19: too little, too late?. Lancet (London, England), 2020; 395(10226): 755. (doi: 10.1016/S01406736(20)30522-5). 
9. Li Q, Guan X, Wu P, Wang X, Zhou L, Tong Y, et al. Early transmission dynamics in Wuhan, China, of novel coronavirus-infected pneumonia. New England Journal of Medicine. N Engl J Med 2020; 382: 2387-2389. (doi: 10.1056/NEJMp2004929).

10. Zheng W. Mental health and a novel coronavirus (2019$\mathrm{nCoV}$ ) in China. Journal of affective disorders, 2020; 269: 201-202. (doi: 10.1016/j.jad.2020.03.041).

11. Banerjee D. The COVID-19 outbreak: Crucial role the psychiatrists can play. Asian journal of psychiatry, 2020; 50: 102014. (doi: 10.1016/j.ajp.2020.102014).

12. Zhang WR, Wang K, Yin L, Zhao WF, Xue Q, Peng M, et al. Mental health and psychosocial problems of medical health workers during the COVID-19 epidemic in China. Psychotherapy and psychosomatics, 2020: 1-9. (doi: 10.1159/000507639).

13. Blake $H$, Bermingham $F$, Johnson $G$, Tabner A. Mitigating the Psychological Impact of COVID-19 on Healthcare Workers: A Digital Learning Package. International Journal of Environmental Research and Public Health, 2020; 17(9): 2997. (doi: 10.3390/ijerph17092997).

14. Tanhan A, Yavuz KF, Young JS, Nalbant A, Arslan G, Yıldırım $M$, et al. A Proposed Framework Based on Literature Review of Online Contextual Mental Health Services to Enhance Wellbeing and Address Psychopathology During COVID-19. Electronic Journal of General Medicine 2020; 17(6): em254. (doi: 10.29333/ejgm/8316).

15. Morin CM, Belleville G, Bélanger L, Ivers $H$. The Insomnia Severity Index: psychometric indicators to detect insomnia cases and evaluate treatment response. Sleep, 2011; 34(5): 601-608. (doi: 10.1093/sleep/34.5.601).
16. Wang HX, Wang L, Zhang WR, Xue Q, Peng M, Sun ZC, et al. Effect of Transcranial Alternating Current Stimulation for the Treatment of Chronic Insomnia: A Randomized, Double-Blind, Parallel-Group, Placebo-Controlled Clinical Trial. Psychotherapy and Psychosomatics, 2020; 89(1): 38-47. (doi: 10.1159/000504609).

17. Chen X, Li P, Wang F, Ji G, Miao L, You S. Psychological results of 438 patients with persisting gastroesophageal reflux disease symptoms by symptom checklist 90revised questionnaire. Euroasian journal of hepatogastroenterology, 2017; 7(2): 117-121. (doi: 10.5005/jpjournals-10018-1230).

18. Sonino N, Fava GA. Rehabilitation in endocrine patients: a novel psychosomatic approach. Psychotherapy and psychosomatics, 2007; 76(6): 319-24. (doi: 10.1159/000107558).

19. Wang ZY. Symptom Check List (SCL-90). Shanghai Jingshen Yixue. 1984; 2(2): 68-70. Chinese.

20. Chen SL, Li L. Re-testing reliability, validity and norm applicability of SCL-90. Chin J Nerv Ment Dis, 2003; 29(5): 323-327.

21. Fava GA, McEwen BS, Guidi J, Gostoli S, Offidani E, Sonino N. Clinical characterization of allostatic overload. Psychoneuroendocrinology. 2019; 108: 94-101. (doi: 10.1016/j.psyneuen.2019.05.028).

22. Zhang S, Lu X, Cao Y, Li Y, Li C, Zhang W. Concerns about disease management and psychological stress in SAPHO patients during the COVID-19 epidemic. medRxiv. PostedMay 11,2020.(doi: 10.1101/2020.05.07.20084087).

23. Bao Y, Sun Y, Meng S, Shi J, Lu L. 2019-nCoV epidemic: address mental health care to empower society. The Lancet, 2020; 395(10224): e37-e38. (doi: 10.1016/S01406736(20)30309-3). 